\title{
A New Discrete Integrable System Derived from a Generalized Ablowitz-Ladik Hierarchy and Its Darboux Transformation
}

\author{
Xianbin Wu, ${ }^{1}$ Weiguo Rui, ${ }^{2}$ and Xiaochun Hong ${ }^{3}$ \\ ${ }^{1}$ Junior College, Zhejiang Wanli University, Ningbo 315100, China \\ ${ }^{2}$ College of Mathematics, Honghe University, Mengzi 661100, China \\ ${ }^{3}$ College of Statistics and Mathematics, Yunnan University of Finance and Economics, Yunnan, \\ Kunming 650221, China
}

Correspondence should be addressed to Weiguo Rui, weiguorhhu@yahoo.com.cn

Received 9 October 2011; Accepted 4 December 2011

Academic Editor: Zuo Nong Zhu

Copyright (C) 2012 Xianbin Wu et al. This is an open access article distributed under the Creative Commons Attribution License, which permits unrestricted use, distribution, and reproduction in any medium, provided the original work is properly cited.

We find an interesting phenomenon that the discrete system appearing in a reference can be reduced to the old integrable system given by Merola, Ragnisco, and Tu in another reference. Differing from the works appearing in the above two references, a new discrete integrable system is obtained by the generalized Ablowitz-Ladik hierarchy; the Darboux transformation of this new discrete integrable system is established further. As applications of this Darboux transformation, different kinds of exact solutions of this new system are explicitly given. Investigatingthe properties of these exact solutions, we find that these exact solutions are not pure soliton solutions, but their dynamic characteristics are very interesting.

\section{Introduction}

It is well known that the nonlinear integrable lattice systems have been intensively investigated in many scientific fields such as physics, chemistry, and biology. The scientific researchers are not only interested in their rich mathematical structures, but also their applications in science, such as mathematical physics, numerical analysis, computer science, statistical physics, and quantum physics. Various integrable lattice systems have been studied extensively, such as the Toda lattice and the relativistic Toda lattice, the Ablowitz-Ladik lattice, the Volterra lattice, the Suris lattice, and some discrete classic equation such as discrete $\mathrm{KdV}$ equation, discrete Schrödinger equation, [1-18].

Recently, some new lattice hierarchies and their integrability, Darboux transformation, conversation law, exact solution, and dynamic characteristics have been holding more and more attention. In addition, the scheme for constructing nonlinear discrete integrable 
couplings [19] and new transformation methods which are used to directly solve discrete integrable systems [20] are also worth paying attention to. Among the multitudinous new integrable hierarchies, we will discuss the so-called new discrete lattice system which is given by [21, equation (2.12)]

$$
q_{n, t}=q_{n}^{2} r_{n+1}-q_{n-1}, \quad r_{n, t}=-r_{n}^{2} q_{n-1}+r_{n+1},
$$

which is derived by Qin. It is very interesting that this equation can be reduced to the old integrable lattice system under a series of local coordinate transformation, which is given by Merola et al. [22]. To see this, we give the whole transformation procedure as follows.

Taking the local coordinate transformation $r_{n+1} \rightarrow \tilde{r}_{n},(1.1)$ can be rewritten as

$$
q_{n, t}=q_{n}^{2} \widetilde{r}_{n}-q_{n-1}, \quad \tilde{r}_{n, t}=-\tilde{r}_{n}^{2} q_{n}+\tilde{r}_{n+1}
$$

Taking another local coordinate transformation $n \rightarrow-\bar{n},(1.2)$ can be rewritten as

$$
q_{-\bar{n}, t}=q_{-\bar{n}}^{2} \tilde{r}_{-\bar{n}}-q_{-\bar{n}-1}, \quad \tilde{r}_{-\bar{n}, t}=-\tilde{r}_{-\bar{n}}^{2} q_{-\bar{n}}+\tilde{r}_{-\bar{n}+1} .
$$

Taking the third coordinate transformation $-\bar{n} \rightarrow \widehat{n}$ and $-(\bar{n}+1) \rightarrow \widehat{n}+1,-(\bar{n}-1) \rightarrow \widehat{n}-1$, (1.3) can be rewritten as

$$
q_{\widehat{n}, t}=q_{\hat{n}}^{2} \tilde{r}_{\widehat{n}}-q_{\widehat{n}+1}, \quad \tilde{r}_{\hat{n}, t}=-\tilde{r}_{\widehat{n}}^{2} q_{\hat{n}}+\tilde{r}_{\hat{n}-1}
$$

To simplify notation, we still use $n$ and $r_{n}$ to take the place of $\hat{n}$ and $\tilde{r}_{n}$. Thus, (1.4) can be rewritten as

$$
q_{n, t}=q_{n}^{2} r_{n}-q_{n+1}, \quad r_{n, t}=-q_{n} r_{n}^{2}+r_{n-1}
$$

Equation (1.5) is equivalent to the following Equation:

$$
\left(\begin{array}{c}
|q\rangle \\
|r\rangle
\end{array}\right)_{t_{1}}=\left(\begin{array}{c}
(\langle q \mid r\rangle-E)|q\rangle \\
\left(E^{-1}-\langle q \mid r\rangle\right)|r\rangle
\end{array}\right)
$$

where $E$ is a shift operator and $|q\rangle \leftrightarrow q_{n},|r\rangle \leftrightarrow r_{n}, t_{1} \leftrightarrow t$. Equation (1.6) was given in [22] as the 44th equation. The results investigated by Qin in [21] are different from those given in [22]; however, these two systems are equivalent under the above coordinate transformations, noting that the coordinate scales of these two systems are different, and both work are interesting and very useful.

The rest of this paper is organized as follows. In Section 2, based on Qin's work, we will derive an indeed new lattice system from the generalized Ablowitz-Ladik hierarchy and establish its Darboux transformation. In Section 3, under the Darboux transformation, using nonzero or zero constant as seeds, we will obtain different kinds of exact solutions of this new system and discuss their dynamic characteristics. It is worthy of mentioning that the exact solutions of this new lattice system which we will obtain have many particular phenomena; 
they are not pure soliton solutions and their waveforms are very interesting. In Section 4, brief conclusions are given.

\section{New Discrete Lattice System and Its Darboux Transformation}

As in [21], we introduce the following discrete spectral problem:

$$
E \psi_{n}=U_{n}\left(q_{n}, r_{n}, \lambda\right) \psi_{n}
$$

where

$$
U_{n}=\left(\begin{array}{cc}
1+\theta q_{n} r_{n} & \lambda q_{n} \\
\lambda r_{n} & \lambda^{2}
\end{array}\right), \quad \theta=0,1
$$

This is a generalization of the Ablowitz-Ladik spectral problem; its corresponding auxiliary problem is shown as follows:

$$
\psi_{n, t}=V_{n}\left(q_{n}, r_{n}, \lambda\right) \psi_{n}
$$

First, we consider the stationary discrete zero curvature equation

$$
\left(E V_{n}\right) U_{n}-U_{n} V_{n}=O,
$$

where

$$
V_{n}=\left(\begin{array}{cc}
\sum_{j=0}^{\infty} a_{n}^{(j)} \lambda^{-2 j} & \sum_{j=0}^{\infty} b_{n}^{(j)} \lambda^{-2 j+1} \\
\sum_{j=0}^{\infty} c_{n}^{(j)} \lambda^{-2 j+1} & -\sum_{j=0}^{\infty} a_{n}^{(j)} \lambda^{-2 j}
\end{array}\right), \quad O=\left(\begin{array}{cc}
0 & 0 \\
0 & 0
\end{array}\right) .
$$

Substituting (2.2) and (2.5) into (2.4), we obtain a series of recursive formulas as follows:

$$
\begin{gathered}
\left(1+\theta q_{n} r_{n}\right)\left[(E-1) a_{n}^{(j)}\right]+r_{n} b_{n+1}^{(j+1)}-q_{n} c_{n}^{(j+1)}=0, \\
b_{n+1}^{(j+1)}+q_{n}\left[(E+1) a_{n}^{(j)}\right]-\left(1+\theta q_{n} r_{n}\right) b_{n}^{(j)}=0, \\
-c_{n}^{(j+1)}-r_{n}\left[(E+1) a_{n}^{(j)}\right]+\left(1+\theta q_{n} r_{n}\right) c_{n+1}^{(j)}=0, \\
q_{n} c_{n+1}^{(j)}-r_{n} b_{n}^{(j)}-(E-1) a_{n}^{(j)}=0, \\
b_{n+1}^{(0)}=c_{n}^{(0)}=0 .
\end{gathered}
$$

Second, we consider the discrete zero curvature equation

$$
U_{n, t}=\left(E V_{n}^{(m)}\right) U_{n}-U_{n} V_{n}^{(m)}
$$


with

$$
V_{n}^{(m)}=\left(\lambda^{2 m} V_{n}^{+}\right)+\Delta_{n}^{(m)}
$$

where the positive power term $V_{n}^{+}$and modification term $\Delta_{n}^{(m)}$ are, respectively, given by

$$
V_{n}^{+}=\left(\begin{array}{cc}
\sum_{j=0}^{m} a_{n}^{(j)} \lambda^{-2 j} & \sum_{j=0}^{m} b_{n}^{(j)} \lambda^{-2 j+1} \\
\sum_{j=0}^{m} c_{n}^{(j)} \lambda^{-2 j+1} & -\sum_{j=0}^{m} a_{n}^{(j)} \lambda^{-2 j}
\end{array}\right), \quad \Delta_{n}^{(m)}=\left(\begin{array}{cc}
-(1-\theta) a_{n}^{(m)} & 0 \\
0 & 0
\end{array}\right) .
$$

Substituting $U_{n}, V_{n}^{(m)}$ into the discrete zero curvature equation (2.7) and recurring to the stationary discrete zero curvature equation (2.3) yield

$$
U_{n, t}=\left(E \Delta_{n}^{(m)}\right) U_{n}-U_{n} \Delta_{n}^{(m)}
$$

Thus, we obtain

$$
\begin{aligned}
& q_{n, t_{m}}=-b_{n+1}^{(m+1)}-(1-\theta) q_{n} a_{n+1}^{(m)}, \\
& r_{n, t_{m}}=c_{n}^{(m+1)}+(1-\theta) r_{n} a_{n}^{(m)}, \\
& \theta\left(q_{n} r_{n}\right)_{t_{m}}=\theta\left(1+\theta q_{n} r_{n}\right)\left(a_{n+1}^{(m)}-a_{n}^{(m)}\right), \quad m=0,1,2, \ldots
\end{aligned}
$$

Obviously, when $\theta=0$ and $\theta=1$, all equations in (2.11) satisfy compatibility condition between (2.1) and the following auxiliary spectral problem:

$$
\psi_{n, t}=V_{n}^{(m)} \psi_{n}
$$

When $j=0,1$ and $\theta=1, m=1$, letting $a_{n}^{(0)}=-1 / 2$ and considering the integral constant as zero, by using (2.6) and (2.11), Qin derived (1.1). Moreover, when $j=0,1,2$ and $\theta=1, m=2$, letting $a_{n}^{(0)}=-1 / 2$ and considering the integral constant as zero, by using (2.6) and (2.11) again, we successfully obtain an indeed new lattice system as follows:

$$
\begin{aligned}
& q_{n, t}=q_{n-1}\left(q_{n-1} r_{n}+2 q_{n} r_{n+1}\right)-q_{n}^{2}\left(q_{n} r_{n+1}^{2}-r_{n+2}\right)-q_{n-2}, \\
& r_{n, t}=-r_{n+1}\left(q_{n} r_{n+1}+2 q_{n-1} r_{n}\right)+r_{n}^{2}\left(q_{n-1}^{2} r_{n}-q_{n-2}\right)+r_{n+2} .
\end{aligned}
$$

Obviously, (2.13) is different from equation (2.12) appearing in [21], and their dynamic properties are very different, see the below discussion. 
The Lax pairs of (2.13) are given by $U_{n}$ in (2.1) and $V_{n}^{(2)}$ in (2.12) when $\theta=1, m=2$. The $V_{n}^{(2)}$ is given by

$$
V_{n}^{(2)}=\left(\begin{array}{cc}
a_{n}^{(0)} \lambda^{4}+a_{n}^{(1)} \lambda^{2}+a_{n}^{(2)} & b_{n}^{(0)} \lambda^{5}+b_{n}^{(1)} \lambda^{3}+b_{n}^{(2)} \lambda \\
c_{n}^{(0)} \lambda^{5}+c_{n}^{(1)} \lambda^{3}+c_{n}^{(2)} \lambda & -a_{n}^{(0)} \lambda^{4}-a_{n}^{(1)} \lambda^{2}-a_{n}^{(2)}
\end{array}\right) .
$$

By using (2.6), when $j=0,1,2$, substituting the values $a_{n}^{(j)}, b_{n}^{(j)}, c_{n}^{(j)}$ into the above matrix and comparing the coefficients of the different power of $\lambda$, we rewrite the matrix $V_{n}^{(2)}$ as

$$
V_{n}^{(2)}=V_{4 n} \lambda^{4}+V_{3 n} \lambda^{3}+V_{2 n} \lambda^{2}+V_{1 n} \lambda+V_{0 n}
$$

where

$$
\begin{gathered}
V_{4 n}=\left(\begin{array}{cc}
-\frac{1}{2} & 0 \\
0 & \frac{1}{2}
\end{array}\right), \quad V_{3 n}=\left(\begin{array}{cc}
0 & q_{n-1} \\
r_{n} & 0
\end{array}\right), \quad V_{2 n}=\left(\begin{array}{cc}
q_{n-1} r_{n} & 0 \\
0 & -q_{n-1} r_{n}
\end{array}\right), \\
V_{1 n}=\left(\begin{array}{c}
0 \\
r_{n+1}\left(1+q_{n} r_{n}\right)-r_{n}\left(q_{n} r_{n+1}+q_{n-1} r_{n}\right)
\end{array}\right. \\
V_{0 n}=\left(\begin{array}{cc}
-q_{n-1}^{2} r_{n}^{2}+q_{n-1} r_{n+1}+q_{n-2} r_{n} & 0 \\
0 & q_{n-1}^{2} r_{n}^{2}-q_{n-1} r_{n+1}-q_{n-2} r_{n}
\end{array}\right) .
\end{gathered}
$$

Next, we establish the Darboux transformation of this new discrete lattice system (2.13). Actually, the Darboux transformation is a special gauge transformation

$$
\widetilde{\psi}_{n}=T_{n} \psi_{n}
$$

of the solutions of the Lax pairs (2.1) and (2.12) when $m=2$, where $\widetilde{\psi}_{n}$ must satisfy Lax pairs (2.1) and (2.12) with some $\tilde{U}_{n}$ and $\widetilde{V}_{n}^{(2)}$, that is,

$$
\begin{gathered}
E \widetilde{\psi}_{n}=\tilde{U}_{n} \widetilde{\psi}_{n}, \quad T_{n+1} U_{n}=\tilde{U}_{n} T_{n}, \\
\widetilde{\psi}_{n, t}=\tilde{V}_{n}^{(2)} \widetilde{\psi}_{n}, \quad T_{n, t}+T_{n} V_{n}^{(2)}=\tilde{V}_{n}^{(2)} T_{n} .
\end{gathered}
$$

Meanwhile, it is required that $\tilde{U}_{n}$ and $\tilde{V}_{n}^{(2)}$ have the same forms as $U_{n}$ and $V_{n}^{(2)}$, respectively. Thus, the old potentials $q_{n}, r_{n}$ in $U_{n}$ and $V_{n}^{(2)}$ are mapped into new potentials $\tilde{q}_{n}, \tilde{r}_{n}$ in $\tilde{U}_{n}$ and $\tilde{V}_{n}^{(2)}$. We assume $T_{n}$ has the following form:

$$
T_{n}=\left(\begin{array}{cc}
\lambda^{2}+A_{n} & B_{n} \lambda \\
C_{n} \lambda & \lambda^{2}+D_{n}
\end{array}\right)
$$

where $A_{n}, B_{n}, C_{n}, D_{n}$ are unknown functions which will be determined later. 
Let $y_{n}=\left(y_{n}^{1}, y_{n}^{2}\right)^{T}$ and $z_{n}=\left(z_{n}^{1}, z_{n}^{2}\right)^{T}$ be two linear independent solutions of Lax pairs (2.1) and (2.12), and suppose that

$$
\psi_{n}=\left(\begin{array}{cc}
y_{n}^{1} & z_{n}^{1} \\
y_{n}^{2} & z_{n}^{2}
\end{array}\right)
$$

Substituting (2.21) into (2.17), we obtain

$$
\widetilde{\psi}_{n}=\left(\begin{array}{cc}
\left(\lambda^{2}+A_{n}\right) y_{n}^{1}+B_{n} \lambda y_{n}^{2} & \left(\lambda^{2}+A_{n}\right) z_{n}^{1}+B_{n} \lambda z_{n}^{2} \\
C_{n} \lambda y_{n}^{1}+\left(\lambda^{2}+D_{n}\right) y_{n}^{2} & C_{n} \lambda z_{n}^{1}+\left(\lambda^{2}+D_{n}\right) z_{n}^{2}
\end{array}\right)
$$

We assume that $\lambda_{1}$ and $\lambda_{2}$ are two roots of det $T_{n}(\lambda)=0$. Therefore, when $\lambda=\lambda_{i}(i=1,2)$, the two columns of $T_{n}$ and $\widetilde{\psi}_{n}$ are linear dependent, respectively. Thus, there exist two group of nonzero parameters $\alpha_{i}=\alpha_{i}(n)$ and $\gamma_{i}=\gamma_{i}(n)(i=1,2)$ which satisfy the following equations:

$$
\begin{gathered}
\lambda_{i}^{2}+A_{n}+\alpha_{i} B_{n} \lambda_{\mathrm{i}}=0, \\
C_{n} \lambda_{i}+\alpha_{i}\left(\lambda_{i}^{2}+D_{n}\right)=0 \\
\left(\lambda_{i}^{2}+A_{n}\right) y_{n}^{1}+B_{n} \lambda_{i} y_{n}^{2}=\gamma_{i}\left[\left(\lambda_{i}^{2}+A_{n}\right) z_{n}^{1}+B_{n} \lambda_{i} z_{n}^{2}\right] \\
C_{n} \lambda_{i} y_{n}^{1}+\left(\lambda_{i}^{2}+D_{n}\right) y_{n}^{2}=\gamma_{i}\left[C_{n} \lambda_{i} z_{n}^{1}+\left(\lambda_{i}^{2}+D_{n}\right) z_{n}^{2}\right] .
\end{gathered}
$$

Solving the above equations, it follows that

$$
\begin{gathered}
A_{n}=\frac{\lambda_{1} \lambda_{2}\left(\lambda_{1} \alpha_{2}-\lambda_{2} \alpha_{1}\right)}{\lambda_{1} \alpha_{1}-\lambda_{2} \alpha_{2}}, \quad B_{n}=\frac{\lambda_{2}^{2}-\lambda_{1}^{2}}{\lambda_{1} \alpha_{1}-\lambda_{2} \alpha_{2}}, \\
C_{n}=\frac{\alpha_{1} \alpha_{2}\left(\lambda_{2}^{2}-\lambda_{1}^{2}\right)}{\lambda_{1} \alpha_{2}-\lambda_{2} \alpha_{1}}, \quad D_{n}=\frac{\lambda_{1} \lambda_{2}\left(\lambda_{1} \alpha_{1}-\lambda_{2} \alpha_{2}\right)}{\lambda_{1} \alpha_{2}-\lambda_{2} \alpha_{1}}, \\
\alpha_{i}=\alpha_{i}(n)=\frac{\gamma_{i} z_{n}^{2}-y_{n}^{2}}{\gamma_{i} z_{n}^{1}-y_{n}^{1}}, \quad i=1,2,
\end{gathered}
$$

where parameters $\lambda_{i}$ and $\gamma_{i}$ (note: $\lambda_{1} \neq \lambda_{2}, \gamma_{1} \neq \gamma_{2}$ ) are suitably chosen such that all the denominators in (2.24) and (2.25) are nonzero.

When $\theta=1$, substituting (2.21) into (2.1), it follows,

$$
\begin{array}{cl}
y_{n+1}^{1}=\left(1+q_{n} r_{n}\right) y_{n}^{1}+q_{n} y_{n}^{2} \lambda_{i}, & z_{n+1}^{1}=\left(1+q_{n} r_{n}\right) z_{n}^{1}+q_{n} z_{n}^{2} \lambda_{i} \\
y_{n+1}^{2}=r_{n} y_{n}^{1} \lambda_{i}+y_{n}^{2} \lambda_{i}^{2}, \quad z_{n+1}^{2}=r_{n} z_{n}^{1} \lambda_{i}+z_{n}^{2} \lambda_{i}^{2} .
\end{array}
$$


Discrete Dynamics in Nature and Society

Applying the shift operator $E$ to all expressions in (2.26) once, it follows

$$
\begin{array}{rlrl}
y_{n}^{1}=\left(1+q_{n-1} r_{n-1}\right) y_{n-1}^{1}+q_{n-1} y_{n-1}^{2} \lambda_{i}, & & z_{n}^{1}=\left(1+q_{n-1} r_{n-1}\right) z_{n-1}^{1}+q_{n-1} z_{n-1}^{2} \lambda_{i} \\
y_{n}^{2}=r_{n-1} y_{n-1}^{1} \lambda_{i}+y_{n-1}^{2} \lambda_{i}^{2}, & z_{n}^{2}=r_{n-1} z_{n-1}^{1} \lambda_{i}+z_{n-1}^{2} \lambda_{i}^{2} .
\end{array}
$$

Solving (2.27) yields

$$
\begin{array}{cc}
y_{n-1}^{1}=\lambda_{i}^{-1}\left(y_{n}^{1} \lambda_{i}-q_{n-1} y_{n}^{2}\right), & z_{n-1}^{1}=\lambda_{i}^{-1}\left(z_{n}^{1} \lambda_{i}-q_{n-1} z_{n}^{2}\right), \\
y_{n-1}^{2}=\lambda_{i}^{-2}\left[\left(1+q_{n-1} r_{n-1}\right) y_{n}^{2}-r_{n-1} y_{n}^{1} \lambda_{i}\right], & z_{n-1}^{2}=\lambda_{i}^{-2}\left[\left(1+q_{n-1} r_{n-1}\right) z_{n}^{2}-r_{n-1} z_{n}^{1} \lambda_{i}\right]
\end{array}
$$

By using (2.26)-(2.28), from (2.25), we get

$$
\alpha_{i}(n+1)=\frac{\mu_{i}}{v_{i}}, \quad \alpha_{i}(n-1)=\frac{\beta_{i}}{\omega_{i}}, \quad i=1,2,
$$

where

$$
\begin{gathered}
\mu_{i}=\mu_{i}(n)=r_{n} \lambda_{i}+\alpha_{i} \lambda_{i}^{2}, \quad \nu_{i}=v_{i}(n)=1+q_{n} r_{n}+\alpha_{i} q_{n} \lambda_{i}, \\
\beta_{i}=\beta_{i}(n)=\left(1+q_{n-1} r_{n-1}\right) \alpha_{i}-r_{n-1} \lambda_{i}, \quad \omega_{i}=\omega_{i}(n)=\lambda_{i}^{2}-\alpha_{i} q_{n-1} \lambda_{i} .
\end{gathered}
$$

According to the above analysis, we derive some useful functions in forthcoming computation process as follows:

$$
\begin{gathered}
A_{n+1}=\frac{\lambda_{1} \lambda_{2}\left(\lambda_{1} \mu_{2} v_{1}-\lambda_{2} \mu_{1} v_{2}\right)}{\lambda_{1} \mu_{1} v_{2}-\lambda_{2} \mu_{2} v_{1}}, \quad B_{n+1}=\frac{v_{1} v_{2}\left(\lambda_{2}^{2}-\lambda_{1}^{2}\right)}{\lambda_{1} \mu_{1} v_{2}-\lambda_{2} \mu_{2} v_{1}}, \\
C_{n+1}=\frac{\mu_{1} \mu_{2}\left(\lambda_{2}^{2}-\lambda_{1}^{2}\right)}{\lambda_{1} \mu_{2} v_{1}-\lambda_{2} \mu_{1} v_{2}}, \quad D_{n+1}=\frac{\lambda_{1} \lambda_{2}\left(\lambda_{1} \mu_{1} v_{2}-\lambda_{2} \mu_{2} v_{1}\right)}{\lambda_{1} \mu_{2} v_{1}-\lambda_{2} \mu_{1} v_{2}} \\
A_{n-1}=\frac{\lambda_{1} \lambda_{2}\left(\lambda_{1} \beta_{2} \omega_{1}-\lambda_{2} \beta_{1} \omega_{2}\right)}{\lambda_{1} \beta_{1} \omega_{2}-\lambda_{2} \beta_{2} \omega_{1}}, \quad B_{n-1}=\frac{\omega_{1} \omega_{2}\left(\lambda_{2}^{2}-\lambda_{1}^{2}\right)}{\lambda_{1} \beta_{1} \omega_{2}-\lambda_{2} \beta_{2} \omega_{1}} \\
C_{n-1}=\frac{\beta_{1} \beta_{2}\left(\lambda_{2}^{2}-\lambda_{1}^{2}\right)}{\lambda_{1} \beta_{2} \omega_{1}-\lambda_{2} \beta_{1} \omega_{2}}, \quad D_{n-1}=\frac{\lambda_{1} \lambda_{2}\left(\lambda_{1} \beta_{1} \omega_{2}-\lambda_{2} \beta_{2} \omega_{1}\right)}{\lambda_{1} \beta_{2} \omega_{1}-\lambda_{2} \beta_{1} \omega_{2}} .
\end{gathered}
$$

We know that the matrix $\tilde{U}_{n}$ keeps the same form as $U_{n}$ in the procedure of mapping the old potentials $q_{n}, r_{n}$ in $U_{n}$ into new potentials $\tilde{q}_{n}, \tilde{r}_{n}$ in $\tilde{U}_{n}$. We can suppose that

$$
\tilde{U}_{n}=\left(\begin{array}{cc}
1+\tilde{q}_{n} \tilde{r}_{n} & \lambda \tilde{q}_{n} \\
\lambda \tilde{r}_{n} & \lambda^{2}
\end{array}\right) .
$$


Substituting $T_{n+1}, U_{n}, T_{n}, \tilde{U}_{n}$ into the second formula in (2.18) and comparing the coefficients of the same power of $\lambda$, it follows that

$$
\begin{gathered}
1+\tilde{q}_{n} \tilde{r}_{n}+\tilde{q}_{n} C_{n}=1+q_{n} r_{n}+r_{n} B_{n+1}, \\
A_{n}\left(1+\tilde{q}_{n} \tilde{r}_{n}\right)=A_{n+1}\left(1+q_{n} r_{n}\right), \\
\tilde{q}_{n}=q_{n}+B_{n+1}, \\
\left(1+\tilde{q}_{n} \tilde{r}_{n}\right) B_{n}+\tilde{q}_{n} D_{n}=q_{n} A_{n+1}, \\
\tilde{r}_{n}+C_{n}=r_{n}, \\
\tilde{r}_{n} A_{n}=\left(1+q_{n} r_{n}\right) C_{n+1}+r_{n} D_{n+1}, \\
\tilde{r}_{n} B_{n}+D_{n}=q_{n} C_{n+1}+D_{n+1} .
\end{gathered}
$$

From (2.36) and (2.38), it is easy to find that there exists a transformation which maps the old potentials $q_{n}, r_{n}$ into new ones $\widetilde{q}_{n}, \widetilde{r}_{n}$ as follows:

$$
\tilde{q}_{n}=q_{n}+B_{n+1}, \quad \tilde{r}_{n}=r_{n}-C_{n} .
$$

Respectively, substituting (2.41) into (2.34), (2.35), (2.37), (2.39), and (2.40), we obtain five conditional equations as follows:

$$
\begin{gathered}
\left(q_{n}+B_{n+1}\right)\left(r_{n}-C_{n}\right)+\left(q_{n}+B_{n+1}\right) C_{n}-q_{n} r_{n}-r_{n} B_{n+1}=0, \\
A_{n}\left[1+\left(q_{n}+B_{n+1}\right)\left(r_{n}-C_{n}\right)\right]-A_{n+1}\left(1+q_{n} r_{n}\right)=0, \\
B_{n}\left[1+\left(q_{n}+B_{n+1}\right)\left(r_{n}-C_{n}\right)\right]+\left(q_{n}+B_{n+1}\right) D_{n}-q_{n} A_{n}=0, \\
\left(r_{n}-C_{n}\right) A_{n}-C_{n+1}\left(1+q_{n} r_{n}\right)-r_{n} D_{n+1}=0, \\
\left(r_{n}-C_{n}\right) B_{n}-q_{n} C_{n+1}+D_{n}-D_{n+1}=0 .
\end{gathered}
$$

Substituting (2.24)-(2.25), (2.29)-(2.30), and (2.31)-(2.32) into the above five conditional equations, taking a tedious direct computation or using the software Maple, we can verify all the above five equations are identities.

In fact, we have directly verified that the (2.41) is the Darboux transformation of the spectral problem (2.1) under the gauge transformation (2.17). Thus, the proof process such as those in [21] can be simplified. Next, we need to verify that the matrix $\tilde{V}_{n}^{(2)}$ defined by (2.19) has the same form as $V_{n}^{(2)}$, that is,

$$
\tilde{V}_{n}^{(2)}=\tilde{V}_{4 n} \lambda^{4}+\tilde{V}_{3 n} \lambda^{3}+\tilde{V}_{2 n} \lambda^{2}+\tilde{V}_{1 n} \lambda+\tilde{V}_{0 n}
$$

where all the forms of $\tilde{V}_{4 n}, \tilde{V}_{3 n}, \tilde{V}_{2 n}, \tilde{V}_{1 n}, \tilde{V}_{0 n}$ are the same as the forms of $V_{4 n}, V_{3 n}, V_{2 n}, V_{1 n}$, $V_{0 n}$. The matrices $\tilde{V}_{4 n}, \tilde{V}_{3 n}, \tilde{V}_{2 n}, \tilde{V}_{1 n}, \tilde{V}_{0 n}$ can be obtained as long as we use the $\tilde{q}$ and $\tilde{r}$ to take the place of $q$ and $r$ in the matrices $V_{4 n}, V_{3 n}, V_{2 n}, V_{1 n}, V_{0 n}$, respectively. 
To simplify computation, according to the coefficients of the different power of $\lambda$, we also rewrite the matrices $T_{n}, T_{n, t}$ as

$$
T_{n}=\left(\begin{array}{cc}
\lambda^{2}+A_{n} & B_{n} \lambda \\
C_{n} \lambda & \lambda^{2}+D_{n}
\end{array}\right)=I \lambda^{2}+T_{1 n} \lambda+T_{0 n}
$$

where

$$
\begin{gathered}
I=\left(\begin{array}{ll}
1 & 0 \\
0 & 1
\end{array}\right), \quad T_{1 n}=\left(\begin{array}{cc}
0 & B_{n} \\
C_{n} & 0
\end{array}\right), \quad T_{0 n}=\left(\begin{array}{cc}
A_{n} & 0 \\
0 & D_{n}
\end{array}\right), \\
T_{n, t}=\left(\begin{array}{cc}
A_{n, t} & B_{n, t} \lambda \\
C_{n, t} \lambda & D_{n, t}
\end{array}\right)=T_{1 n, t} \lambda+T_{0 n, t} \\
T_{1 n, t}=\left(\begin{array}{cc}
0 & B_{n, t} \\
C_{n, t} & 0
\end{array}\right), \quad T_{0 n, t}=\left(\begin{array}{cc}
A_{n, t} & 0 \\
0 & D_{n, t}
\end{array}\right) .
\end{gathered}
$$

Substituting $T_{n, t}, V_{n}^{(2)}, \tilde{V}_{n}^{(2)}$ into the second formula in (2.19) yields

$$
\begin{aligned}
\left(T_{1 n, t} \lambda+T_{0 n, t}\right) & +\left(I \lambda^{2}+T_{1 n} \lambda+T_{0 n}\right)\left(V_{4 n} \lambda^{4}+V_{3 n} \lambda^{3}+V_{2 n} \lambda^{2}+V_{1 n} \lambda+V_{0 n}\right) \\
& -\left(\tilde{V}_{4 n} \lambda^{4}+\tilde{V}_{3 n} \lambda^{3}+\tilde{V}_{2 n} \lambda^{2}+\tilde{V}_{1 n} \lambda+\tilde{V}_{0 n}\right)\left(I \lambda^{2}+T_{1 n} \lambda+T_{0 n}\right)=O .
\end{aligned}
$$

Letting the coefficients of the $\lambda^{6}, \lambda^{5}, \lambda^{4}, \lambda^{3}, \lambda^{2}, \lambda^{1}, \lambda^{0}$ be zero, it follows that

$$
\begin{gathered}
V_{4 n}-\tilde{V}_{4 n}=O \\
\left(T_{1 n} V_{4 n}-\tilde{V}_{4 n} T_{1 n}\right)+\left(V_{3 n}-\tilde{V}_{3 n}\right)=O \\
\left(T_{0 n} V_{4 n}-\tilde{V}_{4 n} T_{0 n}\right)+\left(T_{1 n} V_{3 n}-\tilde{V}_{3 n} T_{1 n}\right)+\left(V_{2 n}-\tilde{V}_{2 n}\right)=O, \\
\left(T_{0 n} V_{3 n}-\tilde{V}_{3 n} T_{0 n}\right)+\left(T_{1 n} V_{2 n}-\tilde{V}_{2 n} T_{1 n}\right)+\left(V_{n}-\tilde{V}_{1 n}\right)=O, \\
\left(T_{0 n} V_{2 n}-\tilde{V}_{2 n} T_{0 n}\right)+\left(T_{1 n} V_{1 n}-\tilde{V}_{1 n} T_{1 n}\right)+\left(V_{0 n}-\tilde{V}_{0 n}\right)=O, \\
T_{1 n, t}+\left(T_{0 n} V_{1 n}-\tilde{V}_{1 n} T_{0 n}\right)+\left(T_{1 n} V_{0 n}-\tilde{V}_{0 n} T_{1 n}\right)=O, \\
T_{0 n, t}+\left(T_{0 n} V_{0 n}-\tilde{V}_{0 n} T_{0 n}\right)=O .
\end{gathered}
$$

So (2.48) holds. By dint of (2.41), we can easily verify that (2.49) and (2.50) hold. Under the transformation (2.41), substituting (2.24), (2.30), (2.31), and (2.32) into (2.51) and (2.52), taking a tedious direct computation or using the software Maple, we can verify that $(2.51)$ 
and (2.52) hold. To simplify computation to verify (2.53) and (2.54), we need some other auxiliary conditions:

$$
\begin{gathered}
A_{n, t}=\frac{\lambda_{1} \lambda_{2}\left(\lambda_{1}^{2}-\lambda_{2}^{2}\right)\left(\alpha_{2, t} \alpha_{1}-\alpha_{2} \alpha_{1, t}\right)}{\left(\lambda_{1} \alpha_{1}-\lambda_{2} \alpha_{2}\right)^{2}}, \quad B_{n, t}=\frac{\left(\lambda_{1}^{2}-\lambda_{2}^{2}\right)\left(\lambda_{1} \alpha_{1, t}-\lambda_{2} \alpha_{2, t}\right)}{\left(\lambda_{1} \alpha_{1}-\lambda_{2} \alpha_{2}\right)^{2}}, \\
C_{n, t}=\frac{\left(\lambda_{1}^{2}-\lambda_{2}^{2}\right)\left(-\lambda_{1} \alpha_{2}^{2} \alpha_{1, t}+\lambda_{2} \alpha_{1}^{2} \alpha_{2, t}\right)}{\left(-\lambda_{1} \alpha_{2}+\lambda_{2} \alpha_{1}\right)^{2}}, \quad D_{n, t}=\frac{\lambda_{1} \lambda_{2}\left(\lambda_{1}^{2}-\lambda_{2}^{2}\right)\left(\alpha_{2} \alpha_{1, t}-\alpha_{1} \alpha_{2, t}\right)}{\left(-\lambda_{1} \alpha_{2}+\lambda_{2} \alpha_{1}\right)^{2}}, \\
\alpha_{i, t}=v_{21}\left(\lambda_{i}\right)-2 v_{11}\left(\lambda_{i}\right) \alpha_{i}+v_{12}\left(\lambda_{i}\right) \alpha_{i}^{2},
\end{gathered}
$$

where

$$
\begin{gathered}
v_{11}\left(\lambda_{i}\right)=-\frac{1}{2} \lambda_{i}^{4}+q_{n-1} r_{n} \lambda_{i}^{2}-q_{n-1}^{2} r_{n}^{2}+q_{n-1} r_{n+1}+q_{n-2} r_{n}, \\
v_{12}\left(\lambda_{i}\right)=q_{n-1} \lambda_{i}^{3}+\left[q_{n-2}\left(1+q_{n-1} r_{n-1}\right)-q_{n-1}\left(q_{n-1} r_{n}+q_{n-2} r_{n-1}\right)\right] \lambda_{i}, \\
v_{21}\left(\lambda_{i}\right)=r_{n} \lambda_{i}^{3}+\left[r_{n+1}\left(1+q_{n} r_{n}\right)-r_{n}\left(q_{n} r_{n+1}+q_{n-1} r_{n}\right)\right] \lambda_{i}, \quad i=1,2 .
\end{gathered}
$$

On the other hand,

$$
\operatorname{det} T_{n}=\left(\lambda^{2}\right)^{2}+\left(A_{n}+D_{n}-B_{n} C_{n}\right) \lambda^{2}+A_{n} D_{n}
$$

Thus, we obtain

$$
A_{n}+D_{n}-B_{n} C_{n}=-\left(\lambda_{1}^{2}+\lambda_{2}^{2}\right), \quad A_{n} D_{n}=\lambda_{1}^{2} \lambda_{2}^{2}
$$

and their differentiation with respect to time $t$

$$
A_{n, t}+D_{n, t}=B_{n, t} C_{n}+B_{n} C_{n, t}, \quad A_{n, t} D_{n}+A_{n} D_{n, t}=0
$$

Applying (2.24), (2.30), (2.31), (2.32), (2.41), and (2.55)-(2.60), we can verify that (2.53) and (2.54) hold. Hence, the following theorem holds.

Theorem 2.1. The solutions $\left(q_{n}, r_{n}\right)$ of the discrete lattice system (2.13) are mapped into their new solutions $\left(\widetilde{q}_{n}, \widetilde{r}_{n}\right)$ under the Darboux transformation (2.41).

\section{New Exact Solutions and Their Properties}

In this section, we will construct exact solutions of discrete system (2.13) by using the Darboux transformation (2.41). Obviously, the $q_{n}=r_{n}=0$ are a couple of seed solutions of system (2.13). In addition, all the constants that satisfy conditional equation $q_{n} r_{n}=2 \pm \sqrt{3}$ are seed solutions of system (2.13). Without loss of generality, we only consider two kinds of seed solutions, that is, $q_{n}=r_{n}=0$ and $q_{n}=1, r_{n}=2+\sqrt{3}$. 
Discrete Dynamics in Nature and Society

\subsection{The Exact Solution Obtained by Nonzero Seed and Its Properties}

When $\theta=1$ and $m=2$, substituting the seed solutions $q_{n}=1, r_{n}=2+\sqrt{3}$ into the Lax pairs (2.1) and (2.12), it follows that

$$
E \psi_{n}=\left(\begin{array}{cc}
3+\sqrt{3} & \lambda \\
(2+\sqrt{3}) \lambda & \lambda^{2}
\end{array}\right) \psi_{n,} \quad \psi_{n, t}=\left(\begin{array}{ll}
a_{11} & a_{12} \\
a_{21} & a_{22}
\end{array}\right) \psi_{n},
$$

where

$$
\begin{gathered}
a_{11}=a_{11}(\lambda)=-\frac{1}{2} \lambda^{4}+(2+\sqrt{3}) \lambda^{2}-(3+2 \sqrt{3}), \quad a_{12}=a_{12}(\lambda)=\lambda^{3}-(1+\sqrt{3}) \lambda, \\
a_{21}=a_{21}(\lambda)=(2+\sqrt{3}) \lambda^{3}-(5+3 \sqrt{3}) \lambda, \quad a_{22}=a_{22}(\lambda)=\frac{1}{2} \lambda^{4}-(2+\sqrt{3}) \lambda^{2}+(3+2 \sqrt{3}) .
\end{gathered}
$$

Equation (3.1) has two real linear independent basic solutions as follows:

$$
\begin{aligned}
& y_{n}=\tau_{1}^{n} \exp \left(\frac{a_{22}+a_{11}+\sqrt{\Delta}}{2} t\right)\left(\begin{array}{c}
a_{22}-a_{11}+\sqrt{\Delta} \\
2 a_{12}
\end{array}\right), \\
& z_{n}=\tau_{2}^{n} \exp \left(\frac{a_{22}+a_{11}-\sqrt{\Delta}}{2} t\right)\left(\begin{array}{c}
a_{22}-a_{11}-\sqrt{\Delta} \\
2 a_{12}
\end{array}\right),
\end{aligned}
$$

where

$$
\begin{aligned}
& \tau_{1}=\frac{(2+\sqrt{3})\left(a_{22}-a_{11}+\sqrt{\Delta}\right) \lambda+2 a_{12} \lambda^{2}}{2 a_{12}}, \quad \tau_{2}=\frac{(2+\sqrt{3})\left(a_{22}-a_{11}-\sqrt{\Delta}\right) \lambda+2 a_{12} \lambda^{2}}{2 a_{12}}, \\
& \Delta=\left(a_{22}-a_{11}\right)^{2}+4 a_{12} a_{21}=\left(1+\sqrt{3}-\lambda_{i}^{2}\right)^{2}\left[\left(\lambda_{i}-1\right)^{2}+2+\sqrt{3}\right]\left[\left(\lambda_{i}+1\right)^{2}+2+\sqrt{3}\right]>0 .
\end{aligned}
$$

By using (2.25), we obtain

$$
\alpha_{i}=\frac{2\left[\lambda_{i}^{3}-(1+\sqrt{3}) \lambda_{i}\right]\left(\gamma_{i}-\delta_{i}^{n} e^{\xi_{i} t}\right)}{\gamma_{i}\left[\left(1+\sqrt{3}-\lambda_{i}^{2}\right)\left(3+\sqrt{3}-\lambda_{i}^{2}\right)-\xi_{i}\right]-\delta_{i}^{n}\left[\left(1+\sqrt{3}-\lambda_{i}^{2}\right)\left(3+\sqrt{3}-\lambda_{i}^{2}\right)+\xi_{i}\right] e^{\xi_{i} t}},
$$

where

$$
\begin{aligned}
& \delta_{i}=\frac{\tau_{1}\left(\lambda_{i}\right)}{\tau_{2}\left(\lambda_{i}\right)}=\frac{(2+\sqrt{3})\left[\left(1+\sqrt{3}-\lambda_{i}^{2}\right)\left(3+\sqrt{3}-\lambda_{i}^{2}\right)+\xi_{\mathrm{i}}\right] \lambda_{i}+2\left[\lambda_{i}^{3}-(1+\sqrt{3}) \lambda_{i}\right] \lambda_{i}^{2}}{(2+\sqrt{3})\left[\left(1+\sqrt{3}-\lambda_{i}^{2}\right)\left(3+\sqrt{3}-\lambda_{i}^{2}\right)-\xi_{i}\right] \lambda_{i}+2\left[\lambda_{i}^{3}-(1+\sqrt{3}) \lambda_{i}\right] \lambda_{i}^{2}}, \\
& \xi_{i}=\sqrt{\left(1+\sqrt{3}-\lambda_{i}^{2}\right)^{2}\left[\left(\lambda_{i}-1\right)^{2}+2+\sqrt{3}\right]\left[\left(\lambda_{i}+1\right)^{2}+2+\sqrt{3}\right]}, \quad i=1,2 .
\end{aligned}
$$


Therefore, by using the Darboux transformation (2.41), (2.24), and (2.31), we obtain the new explicit solution of (2.13) as follows:

$$
\begin{gathered}
\tilde{q}_{n}=1+B_{n+1}=1+\frac{v_{1} v_{2}\left(\lambda_{2}^{2}-\lambda_{1}^{2}\right)}{\lambda_{1} \mu_{1} v_{2}-\lambda_{2} \mu_{2} v_{1}}, \\
\tilde{r}_{n}=2+\sqrt{3}-C_{n}=2+\sqrt{3}-\frac{\alpha_{1} \alpha_{2}\left(\lambda_{2}^{2}-\lambda_{1}^{2}\right)}{\lambda_{1} \alpha_{2}-\lambda_{2} \alpha_{1}},
\end{gathered}
$$

where

$$
\mu_{i}=(2+\sqrt{3}) \lambda_{i}+\alpha_{i} \lambda_{i}^{2}, \quad v_{i}=3+\sqrt{3}+\alpha_{i} \lambda_{i}
$$

which are reduced from (2.30); the parameters $\alpha_{i}, i=1,2$ are defined by (3.6) and $\lambda_{i} \neq \pm$ $\sqrt{1+\sqrt{3}}, \lambda_{1} \neq \pm \lambda_{2}$.

Equation (3.8) is a very complicated solution due to (3.6), and it is hard to analyze its dynamical behavior. Same as $\mathrm{Xu}$, we did not know whether the 26thsolution given in [23] was a soliton solution. We also do not know whether the solution (3.8) is a soliton solution if we only estimate the type of solution from its expression. By the further detailed analysis, we know that the solution (3.8) is not a pure soliton solution, its properties and profiles vary accordingly while the parameters vary. In other words, under the different values of parameters, it contains different waveform. However, in [21], treating $n$ as continuous variable, Qin showed that solution (4.4) possesses properties of one-soliton solutions and two-soliton solutions when $\gamma_{1}, \gamma_{2}, \lambda_{1}$, and $\lambda_{2}$ are taken as different parameters, respectively. In fact, the $n$ is a discrete variable, we cannot regard it as a continuous variable. The dynamic properties and profiles of solution would be distortion if we regard the discrete variable $n$ as a continuous variable. Therefore, regarding $n$ as a discrete variable, we will plot graphs of solution (3.8), some new properties and interesting phenomena will be produced. This shows that our analytic work on dynamic properties of exact solution obtained by the Darboux transformation is very different from the Qin's work.

As an example, when $\gamma_{i}(i=1,2)$ are restricted in the region $0<\gamma_{i}<1$, we investigate the dynamical behavior of solution (3.8). The term $\delta_{i}^{n} e^{\xi_{i} t}$ in (3.6) can be rewritten as $\delta_{i}^{n} e^{\xi_{i} t}=$ $e^{n \ln \left(\delta_{i}\right)+\xi_{i} t}$ when $\delta_{i}>0$, but it cannot be rewritten as this form when $\delta_{i}<0$. It is for this reason that solution (3.8) has variety of properties. For example, when $\delta_{1}>0$ and $\delta_{2}>0$ are both held, solution (3.8) has no oscillation behavior. This can be seen in Figure 1.

Figure 1 shows the properties of double-kink soliton and single-kink soliton with singularity for solutions $\tilde{p}_{n}, \widetilde{r}_{n}$, respectively. Figures $1(\mathrm{a})-1(\mathrm{c})$ show a procedure of leftwards moving for discrete double kink soliton. The waveform gradually changes from Figure 1(a) to Figure 1(c). Particulary, when $t=0$, the waveform becomes a static singular kink soliton, which can be seen in Figure 1(b). Figures 1(d)-1(f) also show a procedure of waveform gradually changes for discrete kink wave. The waveform is a discrete single kink soliton when the time $t<0$, which can be seen in Figure 1(d). Particulary, when the time $t=0$, the waveform also becomes a static singular kink soliton, which can be seen in Figure 1(e). When the time $t>0$, the waveform becomes a moving singular kink wave, which can be seen in Figure 1(f). 


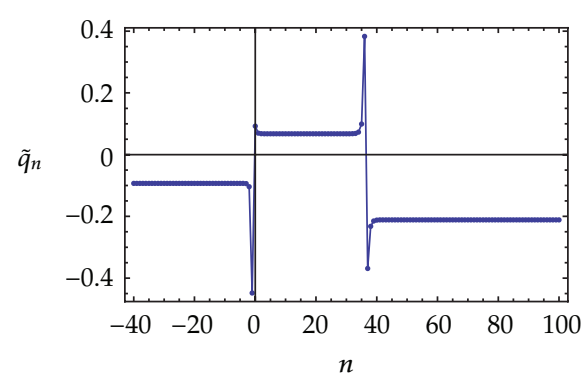

(a) $t=-0.003$

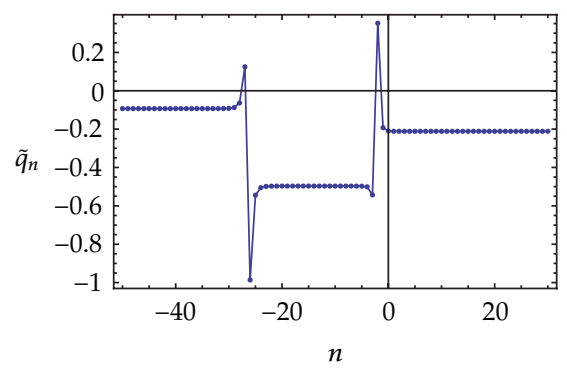

(c) $t=0.002$

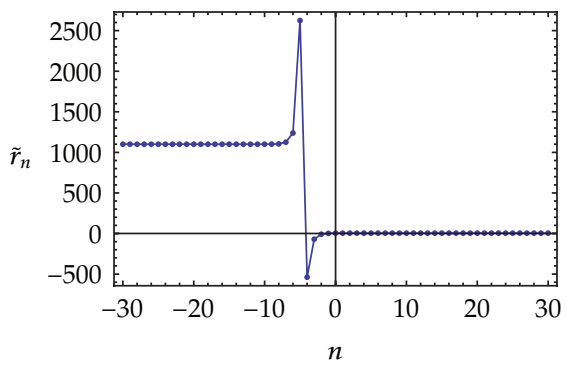

(e) $t=0$

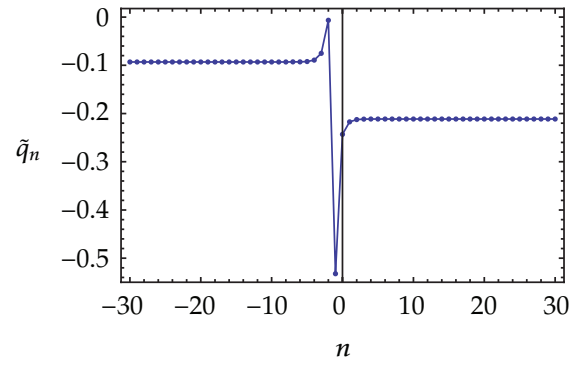

(b) $t=0$

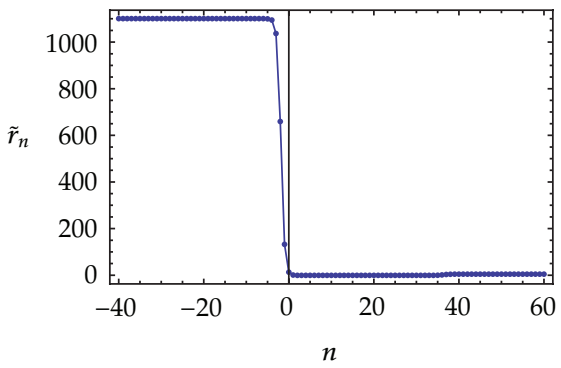

(d) $t=-0.003$

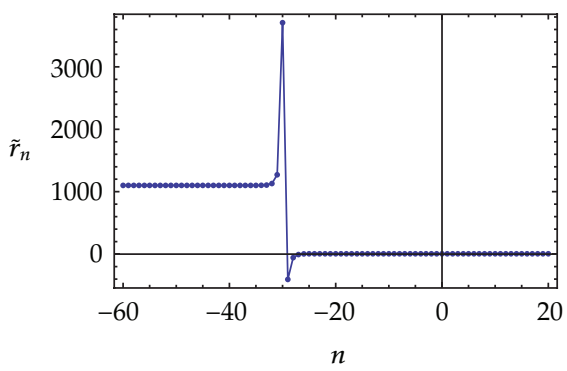

(f) $t=0.002$

Figure 1: The profiles of $\tilde{p}_{n}, \widetilde{r}_{n}$ in (3.8) for $\lambda_{1}=5, \lambda_{2}=12, \gamma_{1}=0.5, \gamma_{2}=0.6, \delta_{1}>0, \delta_{2}>0$.

When both $\delta_{1}<0$ and $\delta_{2}<0$ hold, the solution (3.8) is discrete breather solution; it has breather oscillation behavior shown in Figure 2. In fact, Figure 2 shows the properties of breather soliton with double-kink characteristic for solutions $\widetilde{p}_{n}, \widetilde{r}_{n}$.

Figures 2(a)-2(c) show a procedure of breather oscillation which moves from the right to the left as the time $t$ increases from -2 to 2 . The waveform and amplitude gradually change from Figure 2(a) to Figure 2(c). Figures 2(d)-2(f) also show a procedure of another breather oscillation which moves from the right to the left; the properties and profiles are very similar to those shown in Figures 2(a)-2(c).

However, when $\delta_{1}>0, \delta_{2}<0$ (or $\delta_{1}<0, \delta_{2}>0$ ), solution (3.8) has no breather oscillation behavior anymore. In fact, Figure 3 shows some shapes of singular kink and antikink soliton for solutions $\tilde{p}_{n}, \tilde{r}_{n}$.

Figures 3(a)-3(c) show a procedure of singular antikink soliton which moves from the right to the left as the time $t$ increases from -2 to 3 . The waveform and amplitude gradually change from the form in Figure 3(a) to that in Figure 3(c). Figures 3(d)-3(f) also show a procedure of singular kink soliton which moves from the right to the left; the other properties 


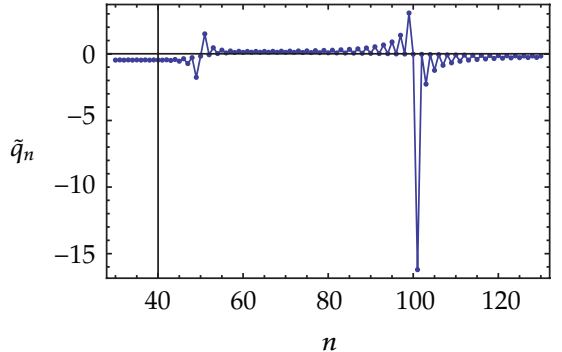

(a) $t=-2$

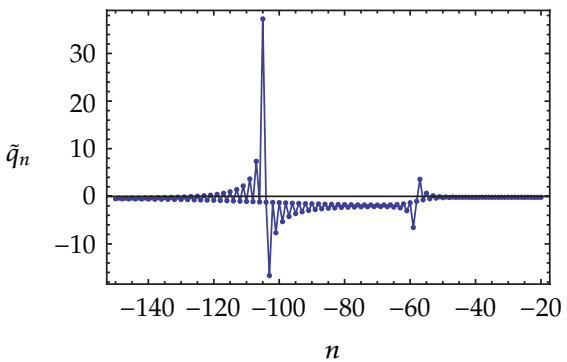

(c) $t=2$

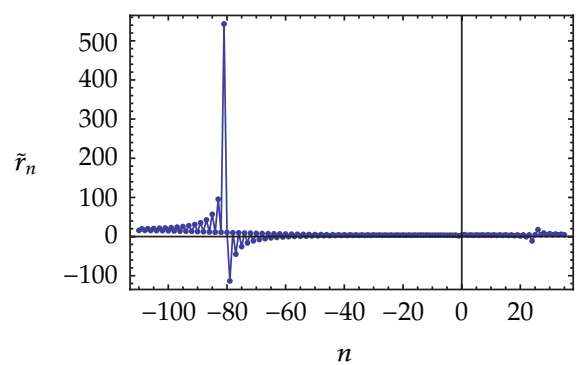

(e) $t=0$

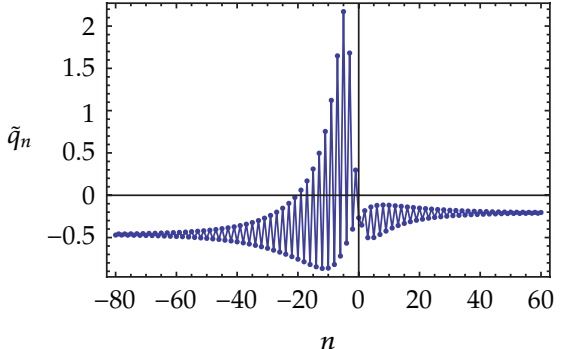

(b) $t=0$

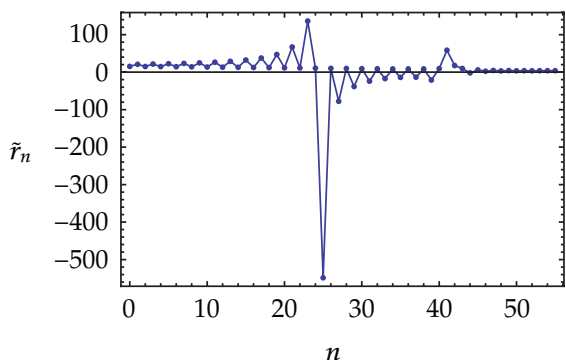

(d) $t=-2$

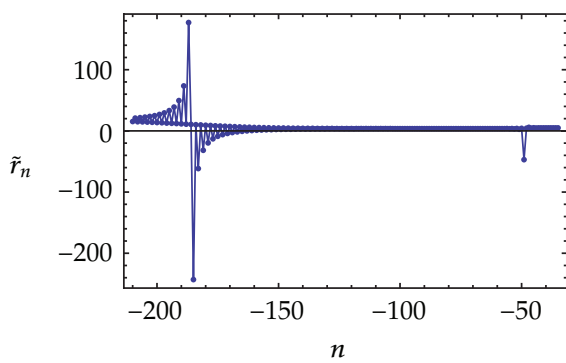

(f) $t=2$

Figure 2: The profiles of $\tilde{p}_{n}, \tilde{r}_{n}$ in (3.8) for $\lambda_{1}=1.8, \lambda_{2}=2, \gamma_{1}=0.5, \gamma_{2}=0.6, \delta_{1}<0, \delta_{2}<0$.

and profiles are very similar to those shown in Figures 3(a)-3(c) except for the static case at the time $t=0$. The waveform in Figure 3(b) has singularity; however, this is not the case in Figure 3(e).

\subsection{The Exact Solution Obtained by Zero Seed and Its Properties}

When $\theta=1$ and $m=2$, substituting the seed solutions $q_{n}=r_{n}=0$ into the Lax pairs (2.1) and (2.12), it follows that

$$
E \psi_{n}=\left(\begin{array}{cc}
1 & 0 \\
0 & \lambda^{2}
\end{array}\right) \psi_{n}, \quad \psi_{n, t}=\left(\begin{array}{cc}
-\frac{1}{2} \lambda^{4} & 0 \\
0 & \frac{1}{2} \lambda^{4}
\end{array}\right) \psi_{n}
$$




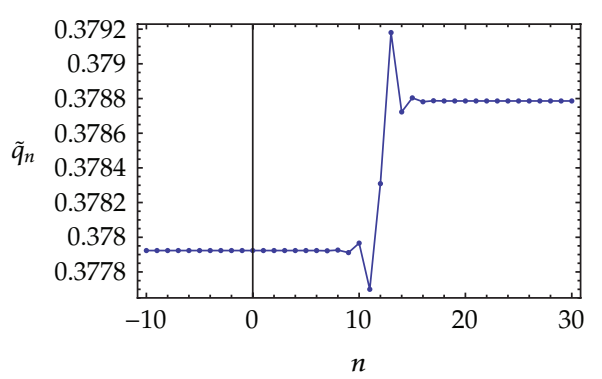

(a) $t=-2$

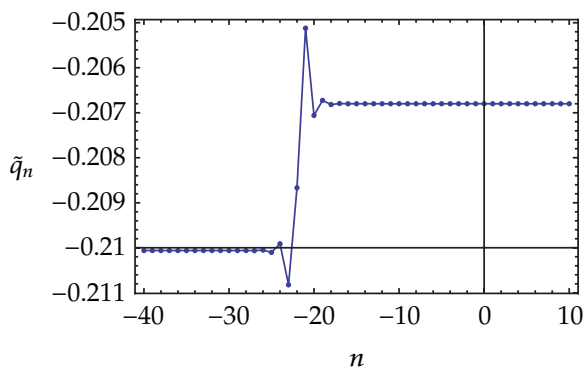

(c) $t=3$

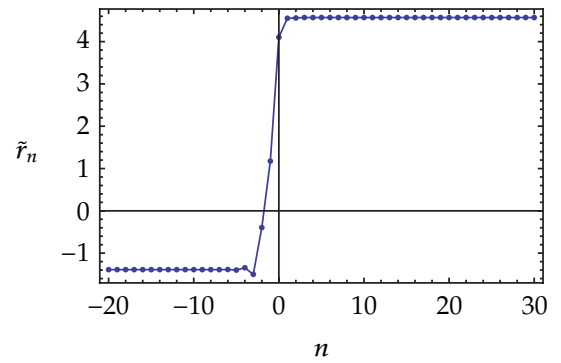

(e) $t=0$

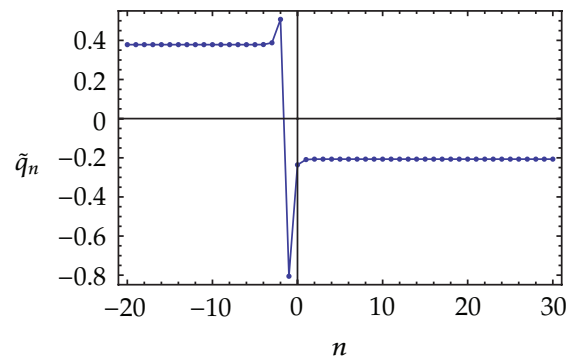

(b) $t=0$

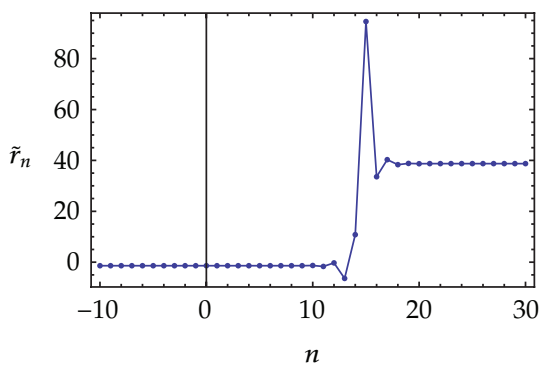

(d) $t=-2$

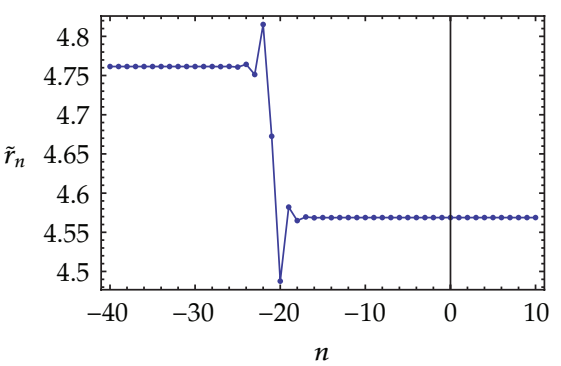

(f) $t=3$

Figure 3: The profiles of $\tilde{p}_{n}, \tilde{r}_{n}$ in (3.8) for $\lambda_{1}=5, \lambda_{2}=1, \gamma_{1}=0.5, \gamma_{2}=0.6, \delta_{1}>0, \delta_{2}<0$.

Equation (3.10) has two real linear independent basic solutions as follows:

$$
\begin{aligned}
& y_{n}=\exp \left(-\frac{1}{2} \lambda^{4} t\right)\left(\begin{array}{l}
1 \\
0
\end{array}\right), \\
& z_{n}=\lambda^{2 n} \exp \left(\frac{1}{2} \lambda^{4} t\right)\left(\begin{array}{l}
0 \\
1
\end{array}\right) .
\end{aligned}
$$

From (3.11), let $y_{n}^{1}=\exp \left((-1 / 2) \lambda^{4} t\right), y_{n}^{2}=0, z_{n}^{1}=0, z_{n}^{2}=\exp \left((1 / 2) \lambda^{4} t\right)$; by using $(2.25)$ and (2.30), we obtain

$$
\alpha_{i}=-\gamma_{i} \lambda_{i}^{2 n} e^{\lambda_{i}^{4} t}, \quad \mu_{i}=\alpha_{i} \lambda_{i}^{2}, \quad v_{i}=1
$$




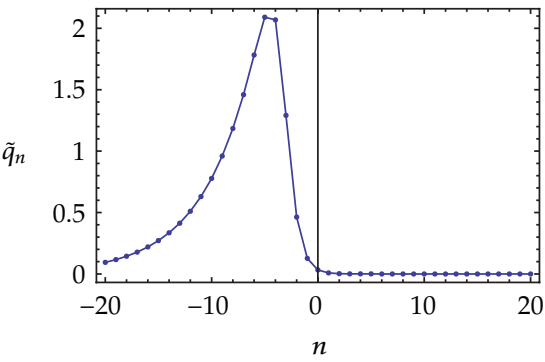

(a) $t=0.2, n \in[-20,20]$

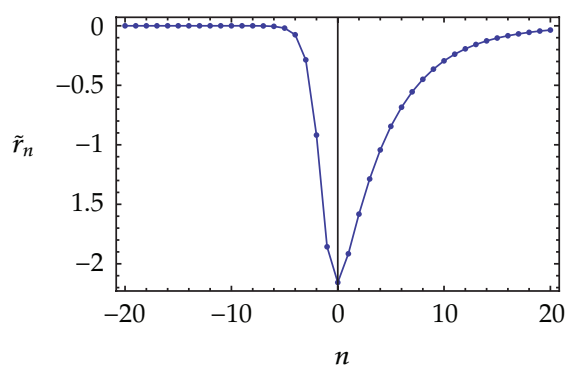

(c) $t=0.2, n \in[-20,20]$

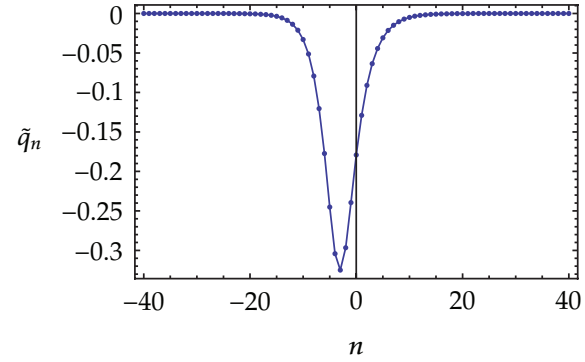

(b) $t=0.2, n \in[-40,40]$

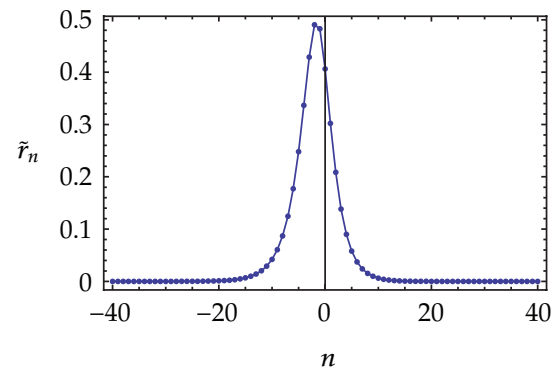

(d) $t=0.2, n \in[-40,40]$

Figure 4: The solutions of $\tilde{p}_{n}, \widetilde{r}_{n}$ in (3.13) show a shape of discrete bright or dark soliton for fixed parametric values. Take (a) and (c) parameters as $\lambda_{1}=2, \lambda_{2}=0.9, \gamma_{1}=0.5, \gamma_{2}=0.6$, and take (b) and (d) parameters as $\lambda_{1}=0.8, \lambda_{2}=-1.2, \quad \gamma_{1}=0.5, \gamma_{2}=1.6$.

Similarly, by using the Darboux transformation (2.41) and (2.24), (2.31), and (3.12), we obtain another explicit solution of (2.13) as follows:

$$
\begin{gathered}
\tilde{q}_{n}=B_{n+1}=\frac{v_{1} \nu_{2}\left(\lambda_{2}^{2}-\lambda_{1}^{2}\right)}{\lambda_{1} \mu_{1} \nu_{2}-\lambda_{2} \mu_{2} \nu_{1}}=\frac{\lambda_{1}^{2}-\lambda_{2}^{2}}{\gamma_{1} \lambda_{1}^{2 n+3} e^{\lambda_{1}^{4} t}-\gamma_{2} \lambda_{2}^{2 n+3} e^{\lambda_{2}^{4} t}}, \\
\tilde{r}_{n}=-C_{n}=-\frac{\alpha_{1} \alpha_{2}\left(\lambda_{2}^{2}-\lambda_{1}^{2}\right)}{\lambda_{1} \alpha_{2}-\lambda_{2} \alpha_{1}}=\frac{\gamma_{1} \gamma_{2}\left(\lambda_{1} \lambda_{2}\right)^{2 n}\left(\lambda_{2}^{2}-\lambda_{1}^{2}\right) e^{\left(\lambda_{1}^{4}+\lambda_{2}^{4}\right) t}}{\lambda_{1} \gamma_{2} \lambda_{2}^{2 n} e^{\lambda_{2}^{4} t}-\lambda_{2} \gamma_{1} \lambda_{1}^{2 n} e^{\lambda_{1}^{4} t}} .
\end{gathered}
$$

In fact, solution (3.13) is not a pure soliton solution for arbitrary parametric values. By the further detailed analysis, we know that solution (3.13) becomes a soliton solution if one of the following parametric conditions holds:

(i) $\lambda_{1}>1,0<\lambda_{2}<1$ or $0<\lambda_{1}<1, \lambda_{2}>1$, and $\gamma_{1} \gamma_{2}<0$;

(ii) $\lambda_{1}<-1,0<\lambda_{2}<1$ or $0<\lambda_{1}<1, \lambda_{2}<-1$ and $\gamma_{1} \gamma_{2}>0$;

(iii) $\left|\lambda_{1}\right|=1,\left|\lambda_{2}\right| \neq 1, \lambda_{1} \lambda_{2}<0$, and $\gamma_{1} \gamma_{2}>0$;

(iv) $\left|\lambda_{1}\right| \neq 1,\left|\lambda_{2}\right|=1, \lambda_{1} \lambda_{2}>0$, and $\gamma_{1} \gamma_{2}<0$.

Under the above parametric conditions (i) or (ii), the solutions $\tilde{p}_{n}, \tilde{r}_{n}$ in (3.13) show discrete bright or dark soliton, which are shown in Figure 4. Under the above parametric 


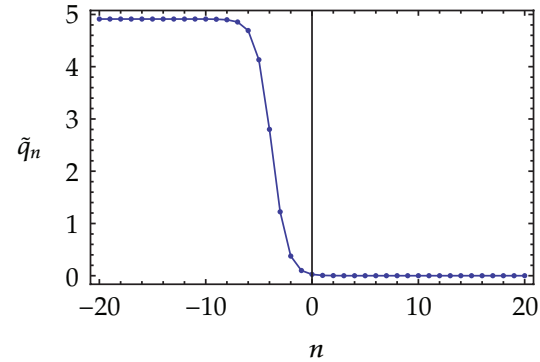

(a) $t=0.2, n \in[-20,20]$

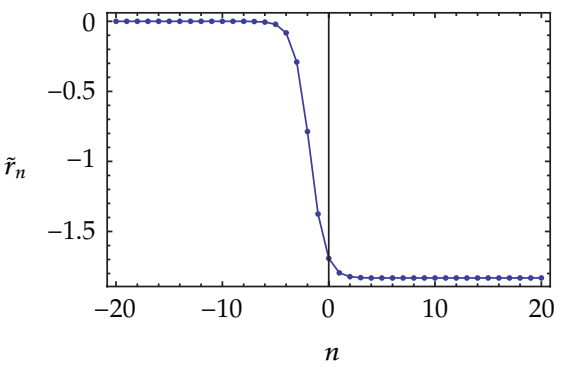

(c) $t=0.2, n \in[-20,20]$

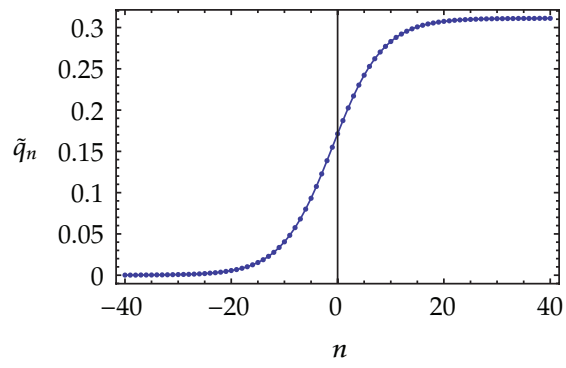

(b) $t=0.2, n \in[-40,40]$

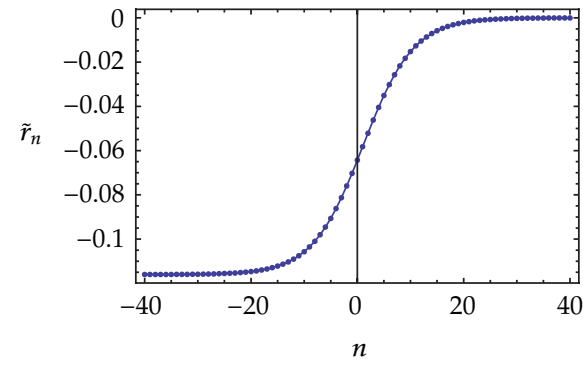

(d) $t=0.2, n \in[-40,40]$

Figure 5: The solutions of $\tilde{p}_{n}, \tilde{r}_{n}$ in (3.13) show a shape of discrete kink or antikink soliton for fixed parametric values. Take (a) and (c) parameters as $\lambda_{1}=1, \lambda_{2}=2, \gamma_{1}=-0.5, \gamma_{2}=0.6$, and take (a) and (c) parameters as $\lambda_{1}=-1, \lambda_{2}=0.9, \gamma_{1}=-0.5, \gamma_{2}=-0.6$.

conditions (iii) or (iv), the solutions $\widetilde{p}_{n}, \widetilde{r}_{n}$ in (3.13) show discrete kink or anti-kink soliton, which are shown in Figure 5.

Under the other parametric conditions, solution (3.13) is not soliton, therefore, we omit those parts of graphs and discussions on dynamic properties.

\section{Conclusions}

In this paper, we show that the discrete system (1.1) which is given by Qin in [21] can be reduced to the old integrable lattice system which is given by Merola et al. [22]. Based on the Qin's work, we present an indeed new discrete integrable system (2.13). Its Darboux transformation is established, and two complex exact solutions (3.8) and (3.13) are obtained by this transformation. By the further detailed analysis, we know that neither of these two exact solutions is pure soliton solutions. Their waveforms vary accordingly while the parameters vary. In other words, under different parametric conditions, these kinds of exact solutions show different dynamic behavior and profiles. In addition, by using different seeds, the obtained solutions are different, so are their properties and waveforms. The solutions obtained by nonzero constant seed mainly show the breather oscillation or singular behavior, but the solutions obtained by zero seed mainly show the profiles of discrete bright or dark soliton and kink or antikink soliton. These show that some exact solutions of discrete integrable systems obtained by the Darboux transformation are it is hard to know whether soliton solutions if we estimate only the types of solution from their expression. It is just because this reason that $\mathrm{Xu}$ did not know whether the solution obtained in [23] was a soliton 
solution. Perhaps, as in $[24,25]$, we call these kinds of solutions $\mathrm{N}$-wave solutions or complexitons which is more accurate. Now, under what kind of parametric conditions, can the exact solutions of discrete integrable systems obtained by Darboux transformation show properties of soliton? This is a very interesting problem. We wish more and more researchers pay attention to this enthralling problem in the future works.

\section{Acknowledgments}

The authors thank three reviewers very much for their useful comments and helpful suggestions. This work was financially supported by the Natural Science Foundation of China (Grant no. 11161038). It was also supported by the Natural Science Foundations of Yunnan Province (Grant no. 2011FZ193) and Zhejiang Province (Grant no. Y2111160).

\section{References}

[1] M. Toda, Theory of Nonlinear Lattices, vol. 20 of Springer Series in Solid-State Sciences, Springer, Berlin, Germany, 2nd edition, 1989.

[2] A. V. Mikhailov, "The reduction problem and the inverse scattering method," Physica D, vol. 3, no. 1-2, pp. 73-117, 1981.

[3] M. Bruschi and O. Ragnisco, "Recursion operator and bäcklund transformations for the RuijsenaarsToda lattice," Physics Letters A, vol. 129, no. 1, pp. 21-25, 1988.

[4] M. Bruschi and O. Ragnisco, "Lax representation and complete integrability for the periodic relativistic Toda lattice," Physics Letters A, vol. 134, no. 6, pp. 365-370, 1989.

[5] M. Bruschi and O. Ragnisco, "The periodic relativistic Toda lattice: direct and inverse problem," Inverse Problems, vol. 5, no. 3, article 011, pp. 389-405, 1989.

[6] S. N. M. Ruijsenaars, "Relativistic Toda systems," Communications in Mathematical Physics, vol. 133, no. 2, pp. 217-247, 1990.

[7] F. Magri, M. Pedroni, and J. P. Zubelli, "On the geometry of Darboux transformations for the KP hierarchy and its connection with the discrete KP hierarchy," Communications in Mathematical Physics, vol. 188, no. 2, pp. 305-325, 1997.

[8] M. Boiti, M. Bruschi, F. Pempinelli, and B. Prinari, "A discrete Schrödinger spectral problem and associated evolution equations," Journal of Physics A, vol. 36, no. 1, pp. 139-149, 2003.

[9] D. Levi and L. Martina, "Integrable hierarchies of nonlinear difference-difference equations and symmetries," Journal of Physics A, vol. 34, no. 48, pp. 10357-10368, 2001.

[10] M. J. Ablowitz, D. J. Kaup, A. C. Newell, and H. Segur, "Nonlinear-evolution equations of physical significance," Physical Review Letters, vol. 31, no. 2, pp. 125-127, 1973.

[11] J. Villarroel, S. Chakravarty, and M. J. Ablowitz, “On a $2+1$ Volterra system,” Nonlinearity, vol. 9, no. 5, pp. 1113-1128, 1996.

[12] Z. N. Zhu and H. W. Tam, "Nonisospectral negative Volterra flows and mixed Volterra flows: lax pairs, infinitely many conservation laws and integrable time discretization," Journal of Physics A, vol. 37, no. 9, pp. 3175-3187, 2004.

[13] W.-X. Ma and X. X. Xu, "A modified Toda spectral problem and its hierarchy of bi-Hamiltonian lattice equations," Journal of Physics A, vol. 37, no. 4, pp. 1323-1336, 2004.

[14] W.-X. Ma, X. X. Xu, and Y. Zhang, "Semidirect sums of Lie algebras and discrete integrable couplings," Journal of Mathematical Physics, vol. 47, no. 5, Article ID 053501, 2006.

[15] Y. B. Suris, "New integrable systems related to the relativistic Toda lattice," Journal of Physics A, vol. 30, no. 5, pp. 1745-1761, 1997.

[16] Y. B. Suris, “On some integrable systems related to the Toda lattice,” Journal of Physics A, vol. 30, no. 6, pp. 2235-2249, 1997.

[17] Z. N. Zhu, H. W. Tam, and Q. Ding, "Infinitely many conservation laws for two integrable lattice hierarchies associated with a new discrete Schrödinger spectral problem," Physics Letters A, vol. 310, no. 4, pp. 281-294, 2003.

[18] Z. N. Zhu and W. Xue, "Two new integrable lattice hierarchies associated with a discrete Schrödinger nonisospectral problem and their infinitely many conservation laws," Physics Letters A, vol. 320, no. 5-6, pp. 396-407, 2004. 
[19] W.-X. Ma and Z. N. Zhu, "Constructing nonlinear discrete integrable Hamiltonian couplings," Computers and Mathematics with Applications, vol. 60, no. 9, pp. 2601-2608, 2010.

[20] Z. Wang and W.-X. Ma, "Discrete Jacobi sub-equation method for nonlinear differential-difference equations," Mathematical Methods in the Applied Sciences, vol. 33, no. 12, pp. 1463-1472, 2010.

[21] Z. Y Qin, "A generalized Ablowitz-Ladik hierarchy, multi-Hamiltonian structure and Darboux transformation," Journal of Mathematical Physics, vol. 49, no. 6, Article ID 063505, 2008.

[22] I. Merola, O. Ragnisco, and G. Z. Tu, "A novel hierarchy of integrable lattices," Inverse Problems, vol. 10, no. 6, article 009, pp. 1315-1334, 1994.

[23] X. X. Xu, "Darboux transformation of a coupled lattice soliton equation," Physics Letters A, vol. 362, no. 2-3, pp. 205-211, 2007.

[24] W.-X. Ma and E. Fan, "Linear superposition principle applying to Hirota bilinear equations," Computers and Mathematics with Applications, vol. 61, no. 4, pp. 950-959, 2011.

[25] W.-X. Ma, "Complexiton solutions to integrable equations," Nonlinear Analysis, vol. 63, no. 5-7, pp. e2461-e2471, 2005. 


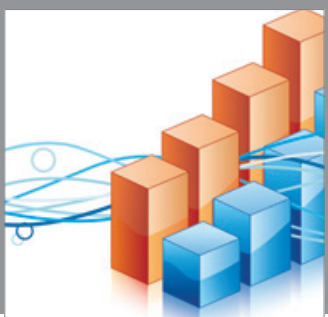

Advances in

Operations Research

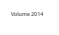

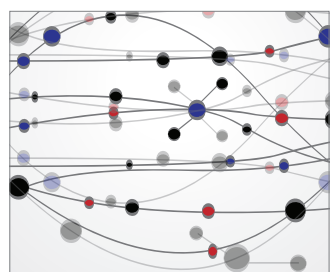

\section{The Scientific} World Journal
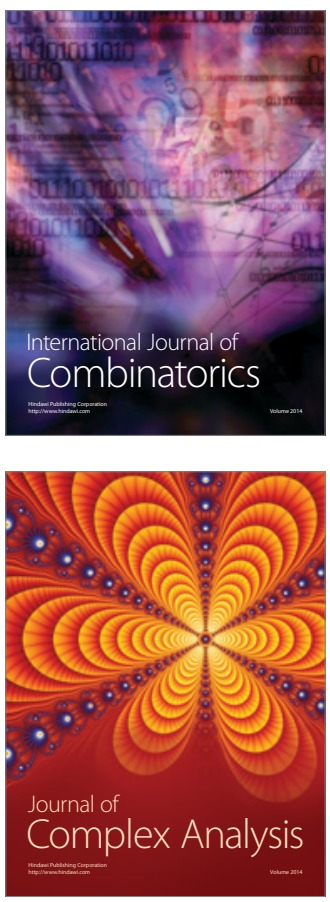

International Journal of

Mathematics and

Mathematical

Sciences
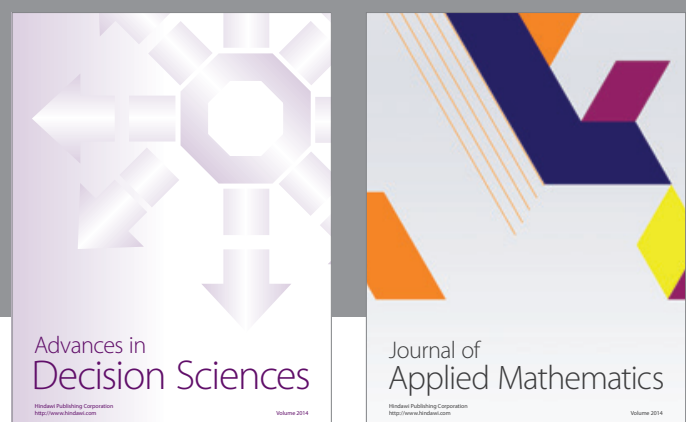

Journal of

Applied Mathematics
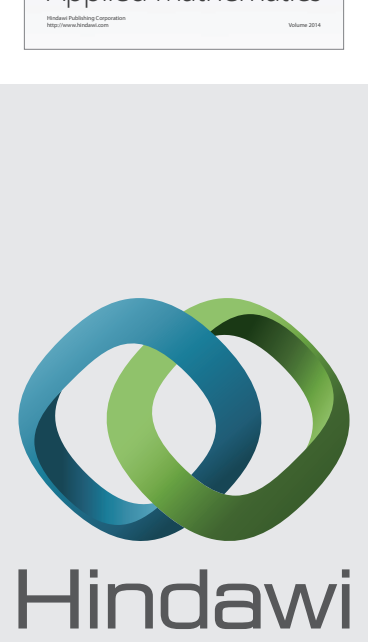

Submit your manuscripts at http://www.hindawi.com
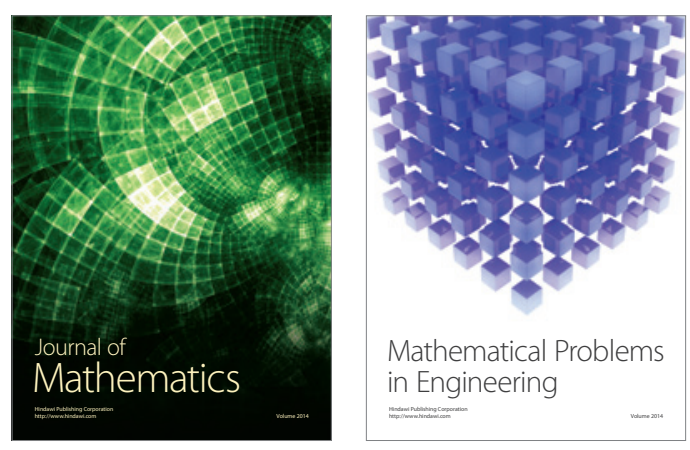

Mathematical Problems in Engineering
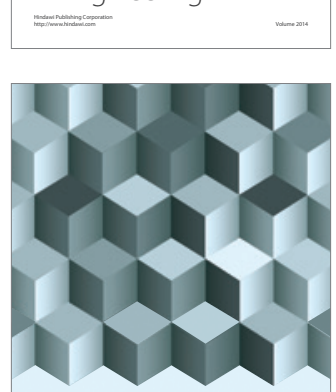

Journal of

Function Spaces
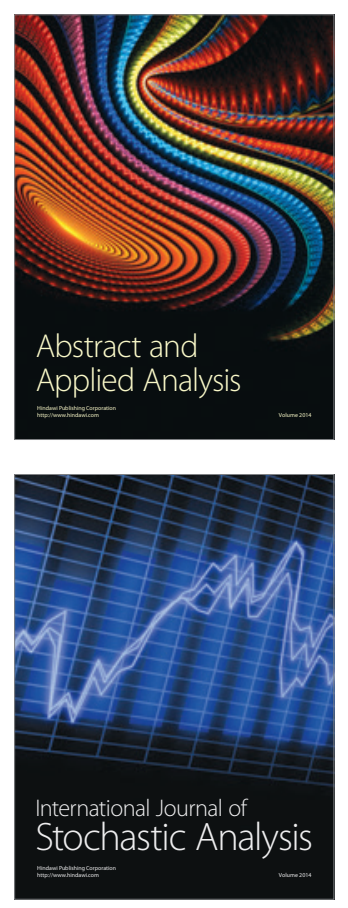

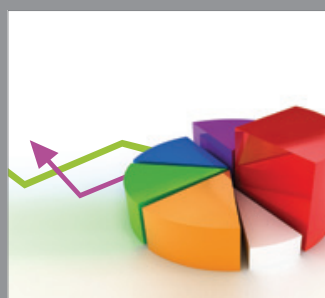

ournal of

Probability and Statistics

Promensencen
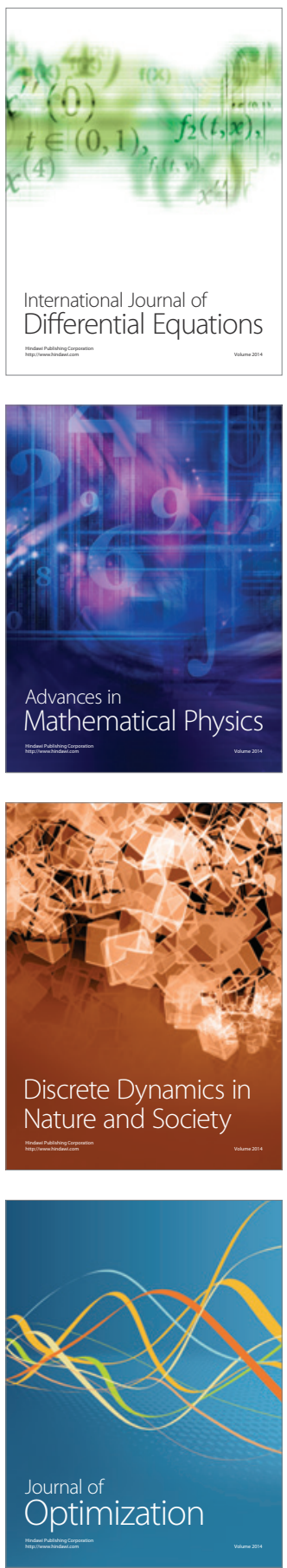